\title{
Remembrance of Places You Passed: Social Spatial Working Memory in Rats
}

\author{
Michael F. Brown, Robert F. Farley, and Edward J. Lorek \\ Villanova University
}

\begin{abstract}
Pairs of rats were tested in a radial-arm maze to determine whether the spatial choices made by one rat affect the subsequent spatial choices of the other rat. In a free-choice procedure, rats showed an increased tendency to choose the location that had most recently been chosen by a foraging partner but a decreased tendency to visit locations that the foraging partner had visited earlier. Forced-choice procedures were used to better control the social stimulus and the interactions between the rats. Under some conditions, locations were chosen later in the choice sequence of a subject rat if another rat had been observed choosing that location. Odor and other physical traces of the other rat's visits were ruled out as explanations for this effect. The results demonstrate the existence of working memory for locations visited by a familiar conspecific.
\end{abstract}

Keywords: animal social behavior, social memory, spatial learning, spatial memory

Although many animals live in relative isolation, others often interact with members of their own species in critically important ways. Given the importance of social interaction, one might expect that the behavior of others often serves as the content of memories and that memory with social content would therefore be an important part of researchers' investigations of animal memory. There is increasing evidence that learning from other individuals is important for many animals (see Galef \& Laland, 2005, for a recent review). However, very little is known about memory for the behavior of other animals.

Many animals forage in groups, including laboratory rats released into a natural habitat (Berdoy, 2002). There are a variety of ideas about the function of social foraging and the variables that affect it (for a review, see Galef \& Giraldeau, 2001). For example, group foraging is generally believed to produce a fundamental trade-off. It provides the benefit of increasing the likelihood that new sources of food will be discovered and/or successfully procured, but at the cost of having to compete with others for access to that food. Many models of social foraging make predictions about the tendency to forage socially and the size of foraging groups on the basis of this trade-off (Giraldeau, 2000). Other theoretical models suggest that animals will adopt different strategies for foraging successfully depending on social conditions. For example, the producer-scrounger model (Barnard \& Sibly, 1981)

Michael F. Brown, Robert F. Farley, and Edward J. Lorek, Department of Psychology, Villanova University.

Experiment 2 served as the basis of a master's thesis submitted to Villanova University by Robert F. Farley. Experiment 3 was supported by a Villanova University Faculty Summer Research Grant to Michael F. Brown. We thank Rachel Bachrach and Tim Grant for assistance conducting these experiments and Haley Solodky for her participation in preliminary work.

Correspondence concerning this article should be addressed to Michael F. Brown, Department of Psychology, Villanova University, 800 Lancaster Avenue, Villanova, PA 19085. E-mail: michael.brown@villanova.edu predicts that animals distribute themselves between two behavioral modes. Some individuals focus their efforts on food procurement ("producers") while others focus on finding other animals who are feeding, thereby locating patches of food ("scroungers"). According to the model, an individual will engage in producer or scrounger behavior depending on the proportion of producers and scroungers currently foraging and the density of food sources in the environment. Other models predict the spatial or temporal distribution of foragers in a group. An influential model of this type is the ideal free distribution (Fretwell \& Lucas, 1970), which predicts that animals will distribute themselves among patches of a resource in proportion to the relative amount (or rate) of the resource available in particular patches. This prediction is based on the fact that the amount of food that can be obtained from a particular patch is a function of the number of foragers currently in the patch, as well as the amount of food in the patch.

These and other well-developed ideas about the ecological factors affecting social foraging clearly suggest the use of cognitive processes to detect, remember, and process information about the behavior of others. Such cognitive processes may be the same ones involved in detecting, remembering, and processing information about physical stimuli, which have been the subject of much empirical and theoretical attention from comparative cognitive psychologists. However, it also seems quite possible that some of the mechanisms involved are adapted specifically for social interaction (e.g., Lefebvre \& Giraldeau, 1996). Surprisingly, there have been very few empirical studies in which the behavior of another animal served as the to-be-remembered stimulus. So although it seems clear that animals must form and use memories of the behavior of others, there is very little systematic evidence that provides confirmation of the existence of memories with social content, let alone empirical analyses of their properties.

An important exception to the lack of knowledge regarding social aspects of animal cognition is the extensive literature on imitative learning (for reviews, see Heyes \& Galef, 1996; Zentall, 2003; Zentall \& Galef, 1988). It is quite clear from this literature 
that animals learn from observing the behavior of other animals, including where food is located (e.g., McQuoid \& Galef, 1992), which food items are palatable (e.g., Galef, 1989; Laland \& Plotkin, 1993), and behaviors used to obtain food (e.g., Laland \& Plotkin, 1990; Lefebvre, Whittle, \& Finkelstein, 1997). It seems likely that such imitative learning involves memory for the observed behaviors (but see Zentall's [2003] analysis of data presented by Gallese, Fadiga, Fogassi, \& Rizzolatti, 1996). The work on imitative learning has focused on the conditions that produce imitation and the nature of the learning process(es) involved, but there has been little or no analysis of the content or mechanism of memories involved in imitation.

In addition, Galef and his colleagues have shown that food aversions and food preferences are socially transmitted in rats (e.g., Galef, 1989; Galef \& Wigmore, 1983). Recently, they have shown that food preferences acquired socially can be retained for at least 1 month (Galef \& Whiskin, 2003) and are retained despite a variety of intervening food-related experiences (Galef, Lee, \& Whiskin, 2005). These findings clearly suggest the involvement of a long-term memory system that stores information obtained from conspecifics.

A project completed in our laboratory provided an initial hint that the spatial choices of rats were affected by choices made earlier by another rat (Brown, Farley, Solodky, \& Bachrach, 2005). The experiment involved the pole box maze, an experimental paradigm developed in the context of our studies of spatial pattern learning (Brown \& Terrinoni, 1996). Pairs of rats (cage mates) were allowed to forage together in the pole box maze. In this paradigm, rats search for sucrose pellets hidden on top of vertical poles. The relevant finding is that during the early trials in this pole box task, rats preferentially chose the poles that had been visited by their cage mate earlier during the trial. However, during later trials in the experiment, the rats preferentially visited the poles that had not been visited by their cage mate earlier in the trial. Poles already visited by the other rat did not contain a pellet. Thus, it appears that the rats started with a preexperimental tendency to search for food where the other rat was-or recently had been-foraging. But over the course of the experiment the rats learned that no food was to be found in places already visited by the other rat. What is critically important about this for the present purposes is that both of these tendencies suggest that the rats are remembering the locations visited by the other rat in a working memory system that codes the relevant information dynamically during each trial.

These results are intriguing, but they have a number of limitations. First, the behavioral tendencies to visit or avoid visiting poles visited by the other rat may not depend solely on memory. The other rat may still be present in the area of the poles it recently visited; thus, the presence of the other rat (as a stimulus), rather than a memory for its earlier behavior, may be controlling behavior. In this sense, the choices made in the pole box maze may not be discrete from the point of view of a foraging partner. Therefore, the pole box maze is probably not the best choice as a paradigm in which to study spatial memories for earlier choices.

The more commonly used radial-arm maze (Olton \& Samuelson, 1976) seemed like a better choice of spatial paradigm in which to examine social working memories because each choice is of a discrete arm of the maze. In the standard version of this task, rats experience a large number of daily trials in which they forage for food in a maze with a central hub and a number of arms (usually 8 or 12) approximately $1-\mathrm{m}$ long. A small amount of preferred food (in our laboratory, two 45-mg sucrose pellets) is placed at the end of each arm, and the rat is allowed to choose arms until all of the food has been collected. A critical feature of the performance of rats in this spatial task is an exceptional ability to avoid visiting locations that they have already depleted of food, an ability that is based on working memory for spatial locations. Under standard conditions, the controlling stimuli used to discriminate among the locations are the visual extramaze stimuli corresponding to the locations (e.g., Olton, 1978; Olton \& Collison, 1979; Suzuki, Augerinos, \& Black, 1980; Zoladek \& Roberts, 1978).

The ability of rats to perform so accurately in the radial-arm maze task has encouraged the view that this task simulates important features of the natural environment in which rats forage. It may therefore involve a specialized (or particularly effective) spatial memory system adapted for gathering food from small patches that are spatially scattered in familiar territory (Bond, Cook, \& Lamb, 1982; Olton \& Schlosberg, 1978; Timberlake, 2002; Timberlake \& Hoffman, 2002). Given that rats forage socially and that the radial-arm maze may support the cognitive processes involved in this behavior, we were encouraged to examine the possibility that working memory for locations visited by another rat might be revealed more clearly in this paradigm.

\section{Experiment 1}

In the first experiment, pairs of rats were tested in a radialarm maze to determine whether the choices made by one rat affect the choices made by a second rat foraging in the same maze and, if so, whether memory is involved. Preliminary work indicated that standard versions of the radial-arm maze do not accommodate two rats on the same maze arm, as the rats cannot pass each other on the narrow arms. We suspected that a modified apparatus, similar to one that had been used in earlier experiments in our laboratory (e.g., Brown \& Moore, 1997), would allow two rats to visit the same arm simultaneously. The key feature of this modified apparatus is that the arms are constructed of polyvinylchloride (PVC) tubes. Relative to more typical radial mazes, this design feature decreases access to the visual cues known to provide the primary spatial information used by rats to navigate in the maze and code spatial locations (e.g., Brown, Rish, VonCulin, \& Edberg, 1993; Olton, 1978; Suzuki et al., 1980). However, previous work clearly indicates that choices in mazes with enclosed arms are also controlled by visual spatial cues (Brown \& Drew, 1998) as long as visual cues are available from the ends of the maze arms (Brown \& Moore, 1997). More important for present purposes, rats readily pass each other (traveling either in the same direction or in opposite directions) in these enclosed, tubular maze arms.

The rats were first shaped to visit the ends of the arms of the maze. They were then tested in daily trials in an otherwise standard radial-arm maze task with their cage mate present in the maze and also making choices. A second phase of the experiment involved forced-choice tests. At the beginning of each trial, the two rats were allowed to choose from four randomly selected maze arms (the other four were rendered 
inaccessible). After a short delay, during which the maze was rotated relative to spatial cues to ensure that subsequent choices were not controlled by odors left by the rats, the two rats were then allowed to choose from among all eight maze arms. The question of primary interest is whether the choices made by the rats are affected by the previous choices of the other rat.

\section{Method}

Subjects. The subjects were 10 male Sprague-Dawley rats, obtained as weanlings from Harlan Sprague Dawley (Indianapolis, IN). They were approximately 3 months old when the experiment began and were housed in pairs. After each rat's initial weight was determined, the rats were placed on a food-restricted diet of approximately $13 \mathrm{gm}$ per day of standard rodent chow (Product No. 2018; Harlan Teklad, Madison, WI). Growth curves provided by the vendor were used to maintain each rat at $90 \%-95 \%$ of its expected weight (based on its initial weight and the growth curve) by adjusting the daily ration. The rats were maintained in a vivarium with a 12-hr light-dark cycle. Experimental procedures occurred during the dark phase of the cycle. One rat in each pair was marked with a large black spot on its back.

Apparatus. The apparatus was an eight-arm radial maze with enclosed arms. The central arena was $46 \mathrm{~cm}$ in diameter with a $25-\mathrm{cm}$-tall opaque plastic wall. The floor of the maze was formed by a layer of the same bedding material used in the subjects' home cages (Harlan Teklad Sani-Chips). The equally spaced arms of the maze were constructed of 10-cm-diameter PVC tubes that extended $80 \mathrm{~cm}$ from the central arena. A platform constructed of the bottom half of these same tubes extended each maze arm an additional $10 \mathrm{~cm}$. Thus, the ends of the arms were open, allowing visual access to the many visual cues in the $3.61 \mathrm{~m} \times 3.53 \mathrm{~m}$ room in which the apparatus was located. A plastic cup $(4.5 \mathrm{~cm}$ square and $0.9 \mathrm{~cm}$ deep) was mounted at the end of the platform and could be baited with two 45-mg sucrose pellets (Product No. PJFSC000045; P. J. Noyes, Lancaster, NH). The maze was mounted on a stand such that the floor of the maze was elevated 15 $\mathrm{cm}$ above the floor of the room and included a lazy Susan device to allow rotation of the maze.

Plastic inserts could be used to block access to maze arms (see Forced-choice tests section). These inserts were plastic flower pots that could be placed into the arm from the central arena. When inserted, they extended $9.5 \mathrm{~cm}$ into the maze arm. Rats were unable to enter the maze arm beyond that distance when an insert was present in a maze arm. "Sham" inserts, constructed in the same manner except that the bottom of the flower pot was cut off, were used in Experiment 2 as described below.

Preliminary training. The rats were first exposed to the maze and trained to obtain pellets from the ends of the maze arms. Each pair of cage mates was placed in the maze with all eight maze arms baited with two pellets for $20 \mathrm{~min}$ per day (or until all pellets in the food cups had been eaten) for 6 consecutive days. During the first day, pellets were scattered inside each maze arm (approximately eight per arm) and in the periphery of the central arena. If both members of a pair entered arms during a trial, then no pellets were placed in the central arena the next day. If both members of a pair obtained one or more pellets from food cups at the end of the arm, then pellets were placed only in the food cup the following day.
Free-choice tests. Cage mates were tested together in daily trials ( 5 or 6 days per week). Prior to each trial, each food cup was baited with two sucrose pellets. The two rats were placed in the central arena, oriented in the same direction (away from the experimenter's station). Choice of a maze arm was defined by a rat having all four paws in the maze arm. The sequence of choices made by the rats was recorded, with each choice identified by rat. The rats were allowed to make choices until $6 \mathrm{~min}$ had elapsed from placement in the maze, at which point they were removed from the maze. Thirty trials were conducted.

Forced-choice tests. Five additional daily trials were conducted using a forced-choice procedure and rotation of the maze. Before each trial, four maze arms were randomly chosen and rendered inaccessible using the plastic inserts. The remaining maze arms were baited. The rats were then placed in the central arena and allowed to make choices until all four accessible maze arms had been chosen by at least one rat. The rats were then removed and placed in a holding cage for $2 \mathrm{~min}$ while the maze was rotated to a randomly chosen orientation (from among eight evenly spaced orientations corresponding to locations in which maze arms had been located during the earlier experimental phases) and any remaining pellets were removed from the maze (so that no pellets were present later in the trial). The rats were then placed back in the central arena of the maze and allowed to make choices until all four locations not visited prior to maze rotation had been visited by at least one rat (regardless of whether the maze arm rotated into that location had been visited).

\section{Results}

All 10 rats had consistently visited maze arms and ate pellets from the food cups by the end of the preliminary training phase.

Free-choice tests. To determine whether choices made by one rat during the free-choice test trials affected the choices made by its cage mate, we coded each choice made by each rat (among the first eight choices made during the trial) in terms of whether the location had been previously chosen by the focal rat itself earlier in the trial and whether it had been chosen by the other rat earlier in the trial. We had reason to suspect (on the basis of unpublished data and casual observation of rats in these experiments) that the effect of choices by the other rat might depend on how recently the other rat had made the choice. Specifically, the other rat might still be physically present in or near the location of its most recent choice, and this might affect the focal rat differently than when the other rat's choice of a maze location was more remote. Thus, maze locations were categorized according to whether the other rat (a) had not visited that location previously during the trial, (b) was the most recent choice of the other rat, or (c) was a choice made earlier in the other rat's choice sequence.

In addition to coding the choices of the focal rat in terms of previous visits by it and by the other rat, we determined a count of the number of locations existing in each of the six categories ( 2 visit status of focal rat $\times 3$ visit status of other rat) at the time of each choice. These two sets of measures (choices made in each category and maze locations available to be chosen in each category) were determined for each rat during each of three blocks of 10 trials. The mean (over rats) proportions of choices 
in each category and the corresponding proportions of locations available during each trial block are shown in Figure 1. In what follows, tendencies to visit (or avoid) maze arms in each category are defined by a difference between the proportion of

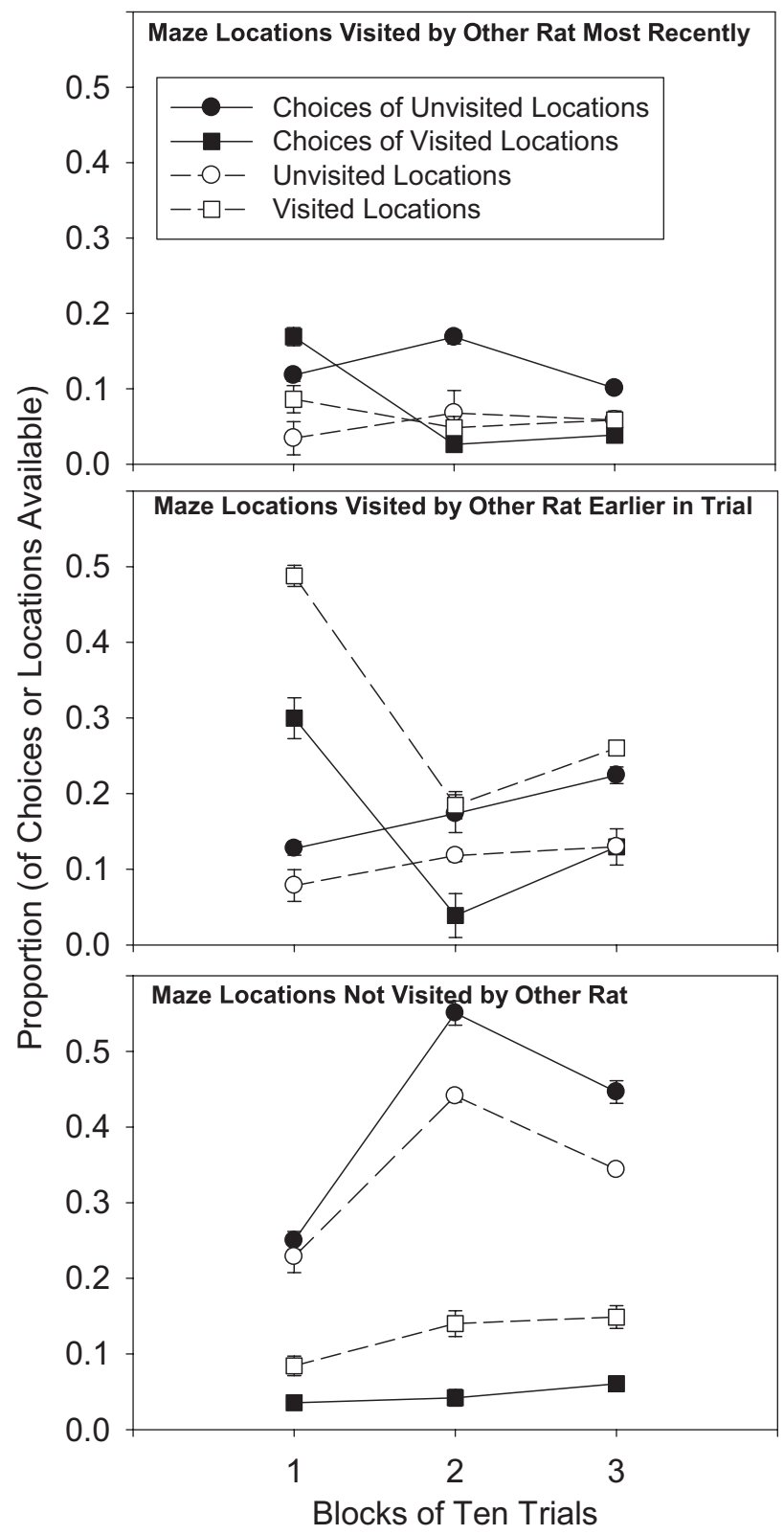

Figure 1. The proportions of maze locations visited and corresponding proportions of maze locations available as choice options during the free-choice trials of Experiment 1. For both locations visited and locations available, proportions are shown as a function of whether the location had been visited by the focal rat earlier during the trial (or not) and whether the location was the most recent choice of the other rat (top panel), had been chosen more remotely during the trials by the other rat (middle panel), or had not been chosen by the other rat (bottom panel). Comparisons of corresponding proportions of choices and locations reveal tendencies to choose or avoid locations as a function of the other rat's choices. Error bars show one standard error of the mean. choices in that category and the corresponding proportion of locations in that category available at the time the choices were made. ${ }^{1}$ In the case of maze locations that had not been chosen by the other rat (bottom panel of Figure 1), there appears to be a higher-than-expected proportion of visits to locations not previously visited by the focal rat and a lower-than-expected proportion of visits to locations that had been previously visited by the focal rat. This is the well-known tendency of rats to avoid revisits of locations in the radial-arm maze.

Because our focus is on the effects of the other rat's visits and because the proportions derived by this analysis are not independent (for each trial block, the proportions of choices in the six categories sum to 1.0 , as do the proportions of locations), our primary analysis of these data was an analysis of variance (ANOVA) that evaluated the proportions of locations visited by the focal rat that had been visited by the other rat (i.e., the data shown in the top and middle panels of Figure 1). The ANOVA compared the proportions of choices with the proportions of locations, along with the factors focal choice (locations previously chosen by focal rat vs. those not previously chosen), other choice (most recent choice of other rat vs. locations chosen more remotely [earlier] during the trial by the other rat), and trial block. Only those factors involving the comparison of proportions of choices and proportions of locations are theoretically meaningful, and so only those factors are reported below. Interactions between the comparison of the two proportions and other factors indicate effects of those factors on the tendency to choose maze locations.

There was an interaction between proportion and other choice, $F(1,9)=133.4$ (the statistical decision criterion used throughout this article is $p<.05)$. The overall proportion of choices made to the location most recently visited by the other rat (.10) was greater than the expected proportion (.06), whereas the overall proportion of choices made to locations visited earlier (i.e., more remotely than the most recent choice; .17) was less than the expected proportion (.21). There was also an interaction between proportion and focal choice, $F(1,9)=$ 105.1. The overall proportion of maze locations that were revisits to locations earlier visited by the focal rat (.12) was less than the expected proportion (.19), and the overall proportion of maze locations that were visits to locations not previously visited by the focal rat (.15) was greater than the expected proportion (.08). There was a significant Proportion $\times$ Focal Choice $\times$ Other Choice interaction, $F(1,9)=101.5$. These effects varied over the course of the experiment, as indicated by the following interactions: Proportion $\times$ Trial Block, $F(2,9)=$ 5.2; Proportion $\times$ Other Choice $\times$ Trial Block, $F(2,18)=11.1$;

\footnotetext{
${ }^{1}$ It should be noted that comparisons of the proportions of choices in these categories with the corresponding proportions of locations available has an important advantage over (simpler) measures that might be used, in particular measures of choices made per opportunity. A rat can choose only one maze location at a time. Thus, when there are large differences in the number of maze locations in the different categories (e.g., at the beginning of a trial, when few locations have been visited, or at the end of a trial, when most locations have been visited), measures based on choices made per opportunity have restricted ranges and complex scalar properties. The measure we used allows direct comparison of the probability of choosing locations in each category with the proportion of choice alternatives in that category at the time choices were made.
} 
Proportion $\times$ Focal Choice $\times$ Trial Block, $F(2,18)=4.4$; and Proportion $\times$ Focal Choice $\times$ Other Choice $\times$ Trial Block, $F(2$, 18) $=8.8$.

A secondary analysis was performed with the specific goal of confirming that earlier visits by the other rat had an effect on the focal rat's choices, beyond the effects of the focal rat's own previous choices. Comparison of the proportions in the middle and bottom panels of Figure 1 suggests that the standard tendency to selectively visit locations that had not been previously visited by the focal rat itself was enhanced when the other rat also had not visited the location and/or reduced when the other rat had visited the location. A second 2 (proportion) $\times 2$ (focal choice) $\times 2$ (other choice) $\times 3$ (trial block) ANOVA, with the levels of other choice being maze locations visited earlier in the trial by the other rat (middle panel of Figure 1) and maze locations not visited by the other rat (bottom panel), was performed to confirm this. The critical Other Choice $\times$ Proportion interaction term was significant, $F(1,9)=107.3$. The form of that interaction was that when the other rat had not visited a location, there was no difference between the mean proportion of choices of that location (.23) and the expected proportion (.23), but when the other rat had visited the location (earlier in its choice sequence than its most recent choice), the proportion of visits by the focal rat (.17) was less than the expected proportion $(.21)$.

Forced-choice tests. Only the initial choice of each location following the trial interruption in the forced-choice test trials was analyzed (i.e., if a rat visited the same location two or more times following rotation of the maze, only the first choice of that location was included). Each choice was categorized in terms of whether it had been visited by the focal rat during the forced-choice phase of the trial (i.e., before maze rotation) and whether it had been visited by the other rat during the forced-choice phase of the trial. A count of the choices in each category was determined for each rat. A count of the number of locations in each of the resulting four categories for each rat was also made, and these parameters were used as numerator and denominator, respectively, to calculate the probability of visits to arms in each category for each rat. Note that the number of locations in each category was fixed within each trial, and so unlike in the analysis of the free-choice tests, comparisons of these probabilities could be made directly. The mean values of these probabilities are shown in Figure 2. As in the free-choice tests (and many other experiments using the radial maze), previous visits by the focal rat decreased the probability of a visit to a location, $F(1,9)=10.4$. More important, previous visits by the cage mate also decreased the probability of a visit to that location, $F(1,9)=12.0$. There was also an interaction between the effects of these two variables, $F(1,9)=4.6$. This interaction can be interpreted in terms of a floor on the probability of visiting arms that had been visited by the focal rat earlier in the trial.

An analogous analysis was carried out in which visits following the trial interruption were coded in terms of the physical maze arm that was rotated into the locations visited following the trial interruption. There was no evidence that the visit status (by either the focal rat or the cage mate) of the maze arm had any effect on the probability of choices made after the trial interruption: all three $F_{\text {s }}(1,9)<1$.

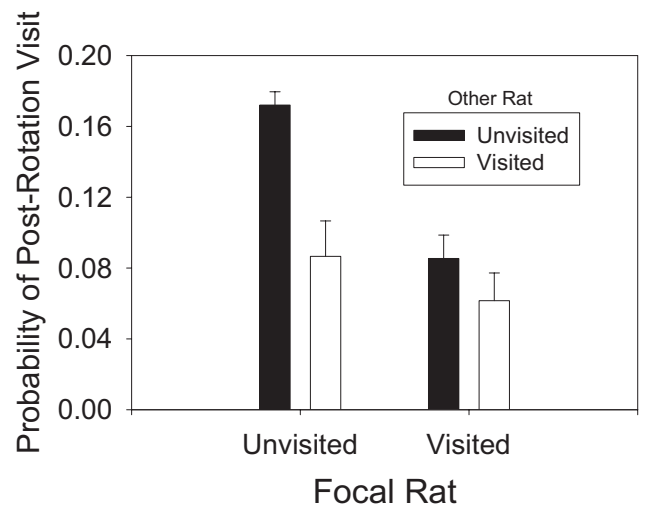

Figure 2. Mean probability of visiting locations during the forced-choice tests of Experiment 1, shown as a function of whether the focal rat itself and the other rat had visited the location prior to trial interruption and maze rotation. Error bars show one standard error of the mean.

\section{Discussion}

The results of this experiment provide clear evidence that the choices made by one rat in a radial maze affect the choices made later in the trial by a second rat. The nature of this effect differs systematically depending on the recency of the second rat's visit. There was a tendency to selectively choose the location that had most recently been visited by another rat. At the same time, there was a tendency to avoid locations that had been visited one or more choices earlier in the other rat's choice sequence. Similarly, there was a tendency to avoid locations that had been visited by the other rat during the forced-choice phase of trials during the subsequent free-choice phase.

This pattern of results indicates that a "following" tendency is elicited by the physical presence of the other rat but that memories for choices made by another rat produce avoidance of those locations. In the free-choice trials, the other rat may still have been present in or near the maze arm it had most recently visited at the time that location was chosen by the focal rat. On the other hand, the other rat would not have been present in, and was less likely to be near, maze arms it had chosen earlier. In the forced-choice trials, our performance measure considers only the effect of choices made by the other rat before the trial interruption on choices made by the focal rat after the trial interruption. Therefore, the physical presence of the other rat could not have been a controlling stimulus in those cases. This indicates that social memories produce avoidance, even though the presence of the other rat is an attraction.

The maze rotation procedure used in the forced-choice tests rules out the possibility that odor cues left by the first rat provide sensory cues necessary for the effect. Thus, as is the case for rats' ability to avoid revisits to maze locations (e.g., Olton \& Collison, 1979), odor cues do not appear to be involved in the ability to discriminate locations visited by a foraging partner. Instead, we infer, memory for the choices made earlier in the trial by the other rat is controlling spatial choices.

\section{Experiment 2}

Experiment 2 was intended to pursue these findings with a larger sample of rats and a technique that allows greater control 
over the interactions between the two rats as well as over the choices made by the other rat. The rats were trained in a freechoice procedure very similar to that used in Experiment 1 . They were then tested in trials in which, during an initial forced-choice phase, only one of the two rats could make choices. During this phase of each trial, the subject rat was prevented from making choices itself but was allowed to observe the other rat as it made choices from a predetermined subset of the locations in the maze. The subject rat then made choices in the absence of the other rat. The primary performance measure allowed us to determine whether the previous choices of the other rat affected the choices made by the subject.

\section{Method}

Subjects. The subjects were 26 experimentally naive male rats from the same source and maintained in the same manner as those in Experiment 1.

Apparatus. The apparatus was the same as that used in Experiment 1 , with one addition. A removable "observation enclosure" was mounted on a pole in the center of the central arena. The enclosure was constructed of a wire mesh basket $(20 \mathrm{~cm}$ in diameter and $14 \mathrm{~cm}$ in height) placed upside down on a clear plastic circular platter. The platter was mounted $6 \mathrm{~cm}$ above the surface of the central arena on a pole that went through the maze to the floor of the laboratory room below. (Although not relevant for Experiment 2, it is important to note for reasons related to Experiment 3 that when the maze was rotated, the observation enclosure remained stationary with respect to the extramaze environment.) The observation enclosure is illustrated in Figure 3. A rat placed inside the enclosure could observe choices being made by another rat in the maze but could not make choices itself.

Preliminary training. Preliminary training was very similar to that used in Experiment 1. The observation enclosure was not present during this or the next phase.

Free-choice tests. Prior to each daily trial, two 45-mg sucrose pellets were placed in each food cup. Each pair of rats was allowed free access to all eight maze arms for 5 min or until all maze

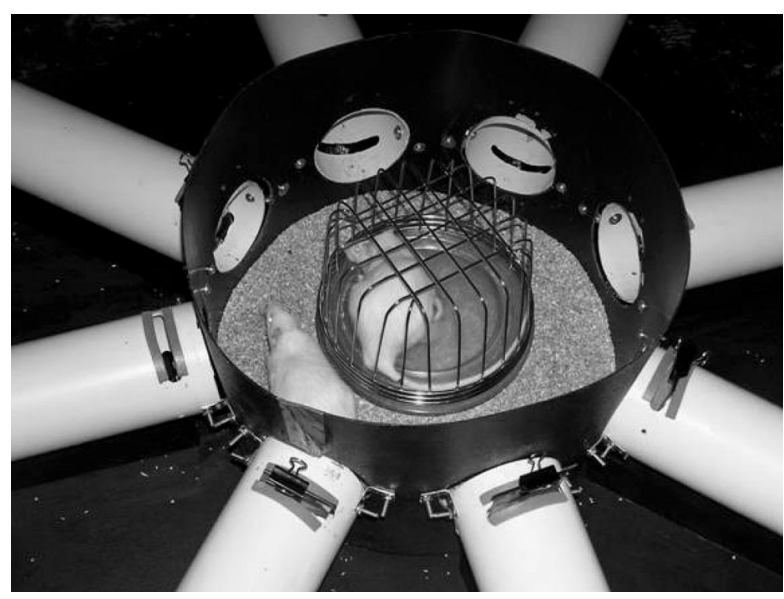

Figure 3. A photograph of the maze, showing the observation enclosure used in Experiments 2 and 3. A subject rat is in the enclosure while a stimulus rat makes choices. locations had been chosen by at least one rat, whichever occurred first. For each trial, the maze was oriented randomly from among the eight possible orientations. Twenty trials were conducted.

Observational testing. Each rat was tested as a subject in 30 trials, structured as six blocks of five trials each. Each rat participated in one trial per day as the subject rat and in a second trial in the role of the "stimulus rat," with the order of roles over the two trials each day randomized. At the beginning of each trial, both rats were placed in the maze, but only one (the stimulus rat) was allowed to make choices. The subject rat was first placed in the observation enclosure. Then the stimulus rat was placed in the central arena and allowed to choose maze arms in a forced-choice procedure in which access to half of the arms (chosen randomly) was blocked by the plastic inserts. Sham inserts were placed in the entrance to each nonblocked arm. The shams appeared similar to the plastic inserts but were cut open at the end facing the outside of the maze to allow rats to pass through to the ends of the maze arms. Insertion of the shams resulted in the appearance of all eight arms being as uniform as possible from the central arena. Once the stimulus rat had chosen all four available arms, it was removed from the maze.

During a 1-min delay, the four inserts and the four sham inserts were removed so that all eight maze arms were accessible. At the end of the 1-min delay, the observation enclosure top (wire mesh basket) surrounding the subject rat was removed. At that point, there were four baited and four unbaited arms, with the unbaited arms corresponding to the previous choices made by the stimulus rat. The subject was allowed to choose freely among the arms until all eight had been chosen or until $5 \mathrm{~min}$ had elapsed since its release from the enclosure, whichever occurred first. While the subject was visiting the maze arm corresponding to its first choice, the bottom (platter) of the observation enclosure was removed, leaving nothing in the central arena except the small pole on which the enclosure had been mounted.

The second trial of the day (during which the rats' roles as subject and stimulus were reversed) always occurred at least 45 min after the first. As in the training phase, the maze was rotated and reoriented randomly for each trial (however, the orientation during the stimulus rat's choices was the same as the orientation during the subject rat's choices).

\section{Results}

Free-choice tests. Performance during free-choice testing was assessed using the same analysis used for the free-choice tests in Experiment 1. The mean proportions of choices made, as well as the corresponding proportions of maze locations available at the time those choices were made, are shown in Figure 4 as a function of whether the focal rat had visited that location earlier during the trial, the visit status of the other rat, and trial block (four blocks of five trials each).

Generally, the pattern of results was very similar to that found in the free-choice trials of Experiment 1. As in Experiment 1, data from locations not visited by the other rat indicated the standard tendency to avoid revisits to locations visited earlier by the focal rat itself (Figure 4, bottom panel), and the primary analysis was an ANOVA performed on the remaining proportions, with only those factors involving the comparison of proportions of choices and proportions of locations reported. There was an interaction be- 


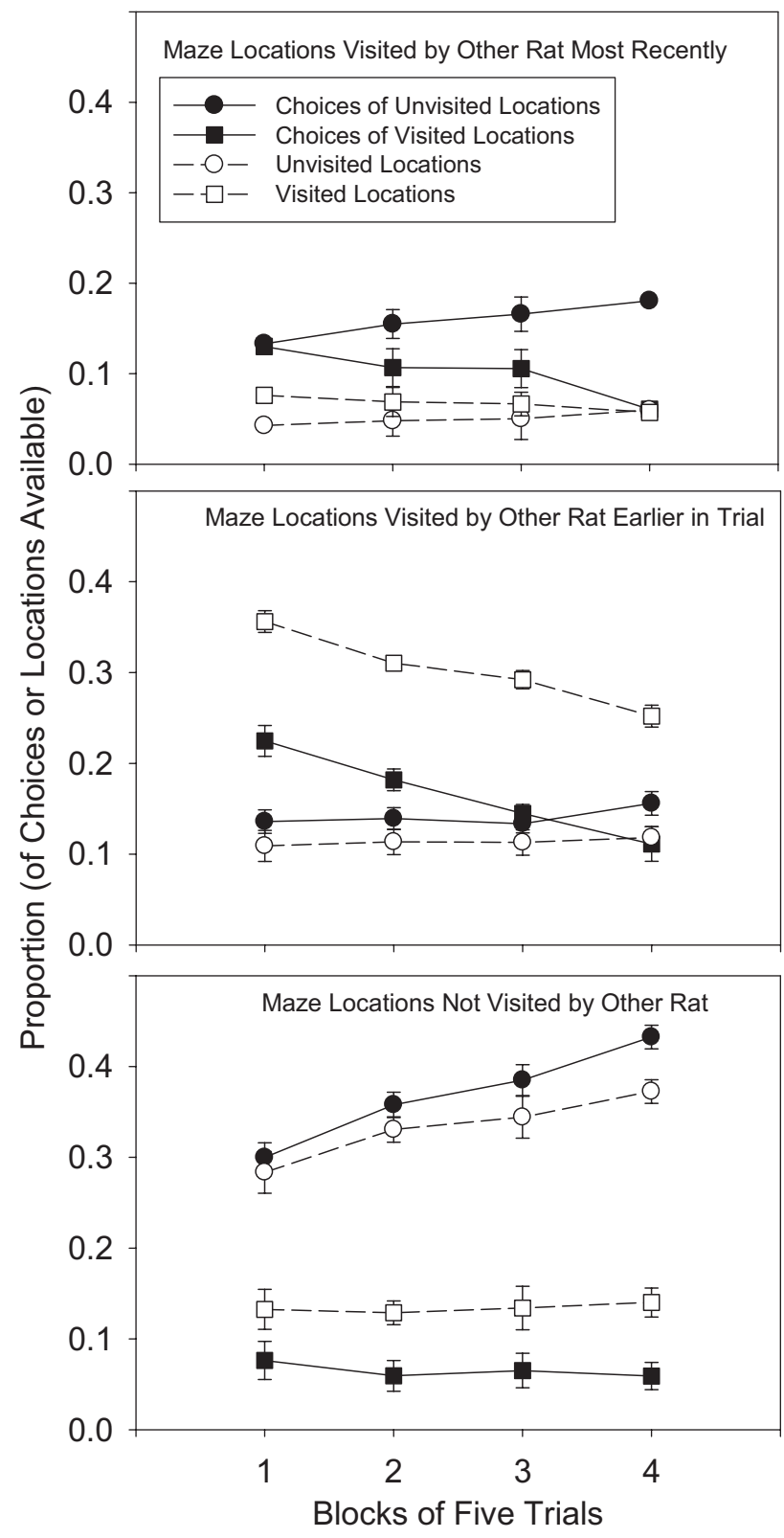

Figure 4. The proportions of maze locations visited and corresponding proportions of maze locations available as choice options during the free-choice trials of Experiment 2. For both locations visited and locations available, proportions are shown as a function of whether the location had been visited by the focal rat earlier during the trial (or not) and whether the location was the most recent choice of the other rat (top panel), had been chosen more remotely during the trials by the other rat (middle panel), or had not been chosen by the other rat (bottom panel). Comparisons of corresponding proportions of choices and locations reveal tendencies to choose or avoid locations as a function of the other rat's choices. Error bars show one standard error of the mean.

tween proportion and other choice, $F(1,25)=58.6$. The overall proportion of choices made to the location most recently visited by the other rat (.13) was greater than the expected proportion (.06), whereas the overall proportion of choices made to locations visited earlier (i.e., more remotely than the most recent choice; .15) was less than the expected proportion (.21). There was also an interaction between proportion and focal choice, $F(1,25)=86.3$. The overall proportion of maze locations that were revisits to locations earlier visited by the focal rat (.13) was less than the expected proportion (.18), and the overall proportion of maze locations that were visits to locations not previously visited by the focal rat (.15) was greater than the expected proportion (.08). The latter effect varied over the course of the experiment: Proportion $\times$ Focal Choice $\times$ Trial Block, $F(3,75)=5.8$. In contrast to the results of Experiment 1, there was no evidence that any other effects were modulated by experience: None of the other interaction terms, including trial block, were significant.

As in Experiment 1, a secondary analysis was performed to confirm that earlier visits by the other rat had an effect on the focal rat's choices beyond the effects of the focal rat's own choices. Comparison of the proportions in the middle and bottom panels of Figure 4 suggests that the standard tendency to avoid revisits to locations that had been previously visited by the focal rat itself was enhanced when the other rat also had visited the location. A second 2 (proportion) $\times 2$ (focal choice) $\times 2$ (other choice) $\times 4$ (trial block) ANOVA, with the levels of other choice being maze locations visited earlier in the trial by the other rat (middle panel of Figure 4) and maze locations not visited by the other rat (bottom panel), was performed to confirm this. The critical Other Choice $\times$ Proportion interaction term was significant, $F(1,25)=34.0$. The form of that interaction was that when the other rat had not visited a location, there was no difference between the mean proportion of choices of that location (.22) and the expected proportion (.23), but when the other rat had visited the location (earlier in its choice sequence than its most recent choice), the proportion of visits by the focal rat (.15) was less than the expected proportion (.21).

Observational testing. Five rats did not transfer smoothly from the free-choice phase of the experiment to the observational testing phase. In 3 cases, rats made few or no choices as stimulus rats, perhaps because of the blocked access to arms. In 2 cases, rats stopped making choices as subject rats. These 5 rats were members of four different pairs of rats. The data from the 8 rats in those four pairs were not included in the analysis.

Among the remaining 18 rats, in the role of subject most chose all eight maze arms within the 5-min limit on most trials (all but one did so on at least 25 trials). Thus, standard measures of which maze locations were chosen would not have allowed discrimination of differences in tendencies to choose location as a function of the stimulus rat's choices. Instead, the primary analysis of the observational test data was designed to determine whether the order in which the subject rat chose maze locations was affected by the choices of the stimulus rat. If the subject rat chose locations without regard to the choices made earlier by the stimulus rat, then the mean serial position of locations in the sequence of choices made by the subject rat should not be affected by whether those locations had been chosen by the stimulus rat. For an eight-arm radial maze, the mean serial position of choices in the sequence of choices is $4.5([1+2+3+4+5+6+7+8] / 8)$, assuming selection without replacement (i.e., ignoring revisits to the same location by the subject). If a subject rat did not choose all eight available arms on a particular trial, then the serial positions of the remaining arms were defined as the mean of the remaining serial positions. For example, if only five of eight arms were chosen, the 
serial position of each of the three remaining locations was coded as 7 , the mean of the remaining serial positions.

Figure 5 shows the mean serial position in the subject's choice sequence of choices made by the stimulus rat. An ANOVA failed to provide evidence that this measure varied over trial blocks, $F(5$, $17)=1.12$. Thus, data from the six blocks were collapsed and compared with the value expected on the basis of chance. The mean serial position of arms visited by the stimulus rat (4.663, $S E M=0.042$ ) was significantly greater than $4.5, t(17)=3.87$, demonstrating that the locations chosen by the stimulus rat were chosen later in the choice sequence of the subject rat.

A secondary analysis was conducted to determine whether the obtained effect was modulated by the sequence in which the stimulus rat initially chose its four available locations. For example, it is possible that only the arm chosen last (out of four) by the stimulus rat affected the choices made by the subject rat. Figure 6 displays the mean serial positions of each of the four arm positions visited by the stimulus rat in the choice sequence of the subject rat. It appears that only the third and fourth arms chosen by the stimulus rat were visited by the subject rat later in its choice sequence. However, an ANOVA failed to reveal a significant effect of serial position on the stimulus rat's choice sequence, $F(3$, 17) $=1.26$

\section{Discussion}

The results of this experiment replicate and extend those of Experiment 1 . In both the free-choice trials and the observational test trials, the choices made by one rat affected the subsequent choices made by another rat. In the free-choice trials, as in Experiment 1 , rats exhibited a tendency to visit the maze location that had been visited most recently by the other rat. However, locations visited by the other rat earlier than its most recent choice were avoided.

In the observational test trials, rats also exhibited a tendency to avoid maze locations that had been visited by the other rat earlier during the trial. In this case, locations visited by the stimulus rat prior to trial interruption were visited relatively later in the choice sequence of the subject rat following trial interruption. The temporal separation between the choices made by the stimulus rat and

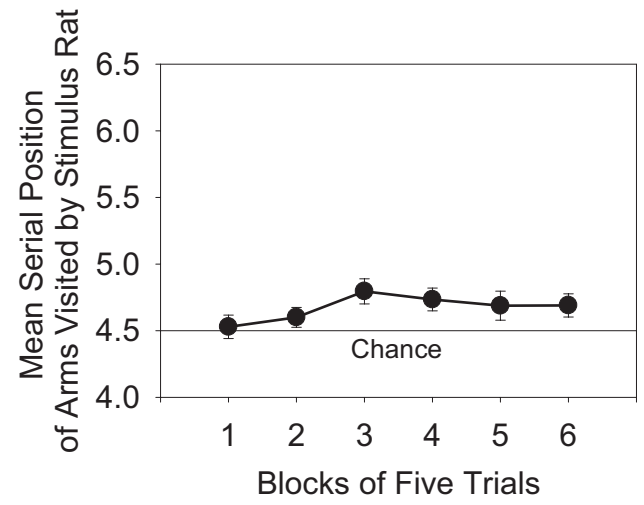

Figure 5. Mean serial position of the locations that had been visited by the stimulus rat in the choice sequence of the subject during the observational tests of Experiment 2. Error bars show one standard error of the mean.

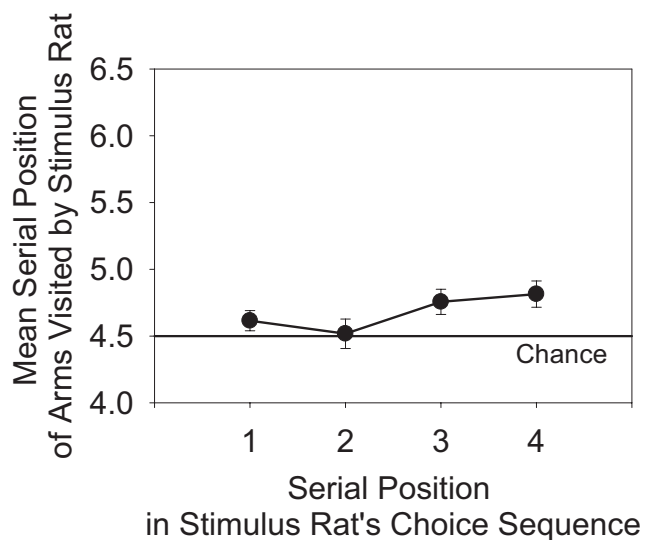

Figure 6. Mean serial position of the locations that had been visited by the stimulus rat in the choice sequence of the subject during the observational tests of Experiment 2. These means are based on the same data shown in Figure 5 but are collapsed over trial blocks and shown as a function of the serial position of the location in the choice sequence of the stimulus rat. Error bars show one standard error of the mean.

the choices made by the subject rat requires memory, unless a physical trace of the stimulus rat's choice persists (Olton \& Collison, 1979). Considering the results of Experiment 2 in isolation, it is possible that odor or some other physical trace of the stimulus rat's choices could be involved, because the maze was not rotated between the stimulus and subject rats' choices. Although this possibility is unlikely given the results of the forced-choice trials in Experiment 1, it is further addressed in Experiment 3.

One might expect that the effect of one rat's choices on those made by another rat would be modulated by the recency of the observed choice event. The tendency of rats to avoid revisits to choices in the radial maze is known to show serial position effects (Cook, Brown, \& Riley, 1985; DiMattia \& Kesner, 1984; Kesner, Chiba, \& Jackson-Smith, 1994). Although the means shown in Figure 6 suggest the possibility that only the most recent choices made by the stimulus rat affected the subject rat's choices, there was no statistical evidence for this effect. However, in the context of the present results, it is important to note that the most recent choice of the stimulus rat did not produce the attractive effect of the most recent visit by the other rat found in the free-choice trials of Experiment 1 and the present experiment. This finding supports the idea that the tendency to visit, rather than avoid, the location most recently visited by the other rat in the free-choice trials resulted from the physical presence of the other rat. When the other rat was not physically present in or near its most recent choice (as was the case in the observation test trials), the focal rat tended not to visit that location.

\section{Experiment 3}

Experiment 3 had two primary purposes. The first was to allow replication of the pattern of results found in the observational test phase of Experiment 2 with a modified experimental procedure that ruled out odor or other intramaze cues as an explanation of discriminating locations chosen by the stimulus rat. The second purpose was to examine whether the contingency between visits to a location by another rat and the presence of food in that location 
determines how visits by the other rat affect spatial choices. Comparisons of win-shift and win-stay contingencies in the standard radial maze task have found an asymmetry in the effects of the contingencies between previous visits of a rat and the presence of food (Bond et al., 1982; Olton \& Schlosberg, 1978). Those comparisons indicate that win-shift contingencies, in which rats find food only in locations not previously visited, result in more accurate performance than win-stay contingencies, in which rats find food only in locations that they have previously visited. More recent work suggests a preexperimental tendency to avoid revisits to maze arms regardless of the reinforcement contingencies (Timberlake \& White, 1990). A primary motivation for the present experiment was our wish to examine whether an analogous asymmetry would be found in the case of locations visited by a foraging partner. We did this by arranging two conditions differing in the contingencies between the stimulus rat's visits and the location of food. In one condition (the different condition), subject rats found food only in the locations that had not been visited by the stimulus rat (as in the earlier experiments). In a second condition (the same condition), subject rats found food only in the same locations that had been visited by the stimulus rat.

Unfortunately, the experiment did not allow a clear comparison of the contingency between arm visits by the other rat and the presence of food in a maze location, because the initial phase of observational testing failed to replicate the basic result found in Experiments 1 and 2. That is, rats did not avoid visits to locations that had been visited by the stimulus rat. Thus, the focus of the experiment changed to determine the basis of this failure to replicate. In particular, rats in the first phase of the experiment had been given minimal exposure to the free-choice procedure in order to allow uncontaminated exposure to the two experimental conditions. Perhaps the lack of exposure to the usual and natural contingency (in which a visit by another rat corresponds to a lack of food in that location) was responsible for the failure to replicate. Motivated by this possibility, we exposed all of the rats in the experiment to free-choice trials like those used in the earlier experiments and then tested them again in observational tests.

An additional feature of this experiment was the use of maze rotation during the trial interruption (between the choices made by the stimulus rat and those made by the subject) to rule out odor or other intramaze stimuli as cues. Thus, any effect of the choices made by the stimulus rat on the subsequent choices of the subject can be explained only in terms of memory for those choices.

\section{Method}

Subjects. The subjects were 20 experimentally naive male rats from the same source and maintained in the same manner as those in Experiments 1 and 2.

Apparatus. The apparatus was the same radial-arm maze used in Experiment 2 except for a change in the mechanism used to prevent access to maze arms during the stimulus rat's forced-choice phase of the trials. The plastic inserts used in the earlier experiments were replaced by clear Plexiglas doors, which could be inserted into slots cut into each maze arm $3 \mathrm{~cm}$ from where the arm was attached to the central arena (the slots for these doors can be seen in Figure 3). The doors could be placed in individual maze arms prior to each trial. They allowed access to the visual cues available at the end of each arm, but they prevented the stimulus rat from choosing or visiting an arm when a door was present.

Preliminary training. Preliminary training was conducted in a manner very similar to that used in Experiment 2. It required four daily sessions and was followed immediately by observational testing.

Observational Testing I. Test trials were conducted just as the observational test trials in Experiment 2, with the following exceptions. The orientation of the maze was randomly chosen from among the eight possibilities prior to each trial and was rotated to a second randomly chosen orientation after the stimulus rat was removed. While the maze was rotated, the subject remained in the observation enclosure, which did not rotate with the maze (it remained stable in relation to the extramaze environment). After being released from the observation enclosure, the subject rat was allowed to make choices until all four baited locations had been chosen or $7 \mathrm{~min}$ had elapsed.

One rat from each pair of cage mates was randomly selected to be in the same condition; the other was assigned to the different condition. The experimental procedures for rats in the two conditions were identical, with one exception. In the different condition, as in Experiments 1 and 2, baited locations had not been visited by the other rat. In the same condition, however, the locations baited when the subject rat made its choices were the locations that had been visited by the stimulus rat (regardless of the identity of the physical maze arm that had been rotated into that location). Thus, after the stimulus rat was removed from the maze, the maze was rotated into its new orientation. Four maze arms were then baited, depending on the condition to which the subject rat was assigned. For samecondition rats, the maze arms in the same four locations visited by the stimulus rat were baited. For different-condition rats, the maze arms in the four locations that had not been visited by the stimulus rat were baited.

Each rat participated in two trials per day, once as the subject rat and once as the stimulus rat. The order in which these two types of trials occurred each day was counterbalanced for each rat. Each rat participated as the subject in 40 trials.

Free-choice training. The rats were next exposed to freechoice trials identical to trials of the free-choice training in Experiment 2. That is, cage mates chose from among eight baited maze arms until all eight arms had been chosen by at least one rat or $5 \mathrm{~min}$ had elapsed. Thus, the rats that had been in the two experimental conditions were now all exposed to a "different" contingency, in that locations previously visited by the other rat did not contain food pellets. Twenty-five daily free-choice training trials occurred.

Observational Testing II. The rats were then returned to a phase in which forced-choice, observational trials were conducted. These trials were structured identically to those in the Observational Testing I phase, except that all 20 rats now found pellets in the locations that had not been visited by the stimulus rat (i.e., all rats were now in the different condition). Each rat participated as a subject in 20 trials (and also served as the stimulus rat for its cage mate during 20 trials). 


\section{Results}

Observational Testing I. The mean serial position of the locations visited by the stimulus rat in the choice sequence of the subject rat was determined in the same manner as in Experiment 2. The mean serial positions for rats in the two conditions are shown (over blocks of 10 trials) in Figure 7. There is no evidence that the choices made by the stimulus rat affected the choices made by the subject. A Trial Block $\times$ Condition ANOVA provided no evidence for a difference in the mean serial position as function of trial block, $F(1,18)<1$, or experimental condition, $F(1,18)=2.79$, $p=.112$, or for an interaction between effects of these variables, $F(1,18)<1$. In addition, $t$ tests comparing the mean serial positions for rats in each experimental condition (collapsed over trial blocks) with the chance value of 4.5 failed to reveal a difference between the mean serial position of choices to locations visited by the stimulus rat and chance performance in either group of subjects, $t \mathrm{~s}(9)<1$.

Observational Testing II. Analogous results from the observational test sessions that followed the free-choice trials are shown in Figure 8. A Trial Block $\times$ Condition ANOVA confirmed that there was a difference between the mean serial position in the subject's choice sequence of locations visited by the stimulus rat, depending on which condition the subject had been exposed to during $\mathrm{Ob}-$ servational Training I, $F(1,18)=21.2$. Mean serial position did not differ over trial blocks, $F(1,18)=1.32$, nor was there an interaction between the effects of these variables, $F(1,18)<1$. Comparisons of the mean serial positions (collapsed over trial blocks) with the chance value of 4.5 showed that the mean serial position of locations visited by the stimulus rat was greater than chance for rats that had been in the different condition $(M=4.72)$, $t(9)=5.32$. Thus, those locations tended to be visited late in the subject rat's choice sequence. The corresponding mean serial position for subjects that had been in the same condition did not differ from chance $(M=4.42), t(9)=1.62$.

\section{Discussion}

No evidence for social effects on choices was found during Observational Testing I. As noted above, at the time of that phase

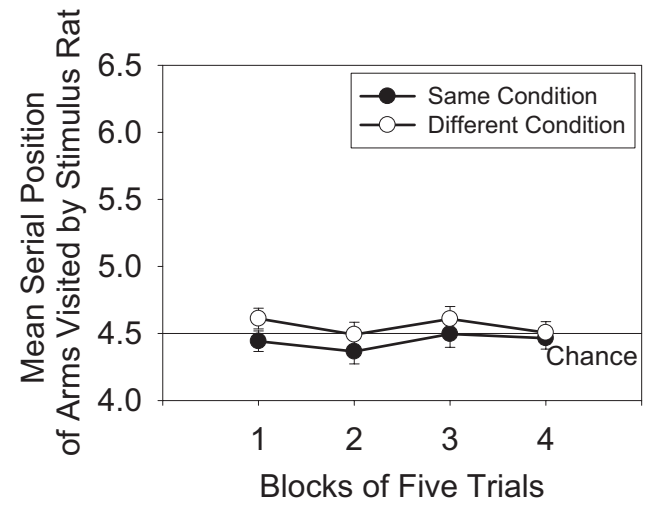

Figure 7. Mean serial position of the locations that had been visited by the stimulus rat in the choice sequence of the subject during the Observational Testing I phase of Experiment 3. Data are shown separately for rats in the same and different experimental conditions. Error bars show one standard error of the mean.

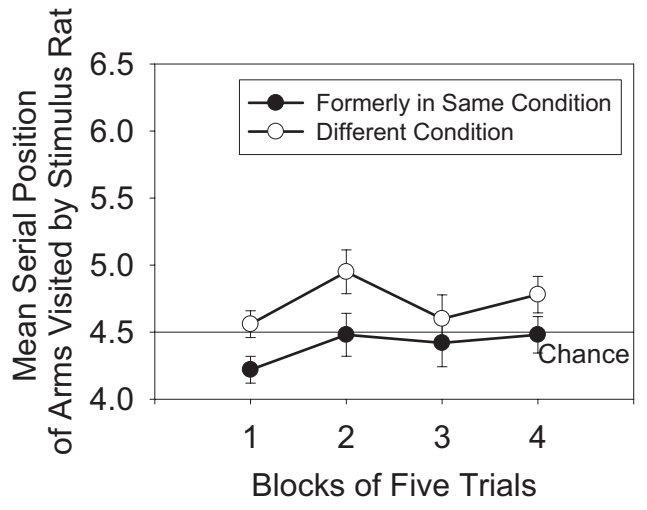

Figure 8. Mean serial position of the locations that had been visited by the stimulus rat in the choice sequence of the subject during the Observational Testing II phase of Experiment 3. Data are shown separately for rats that had been in the same and different experimental conditions during the Observational Testing I phase of the experiment. Error bars show one standard error of the mean.

of the experiment, the rats had only very minimal exposure to free-choice training. They therefore had minimal exposure to the contingency between another rat's visit and the absence of food in that location. This was by design: We wanted to minimize exposure to that contingency among rats in the same condition. In addition, the rats had only minimal interaction in the maze with a foraging partner. In the observation testing procedure, physical contact between the rats is reduced and the ability to "follow" the other rat into a maze arm is prevented. It is possible that one or both of these factors prevented the rats from being affected by the choices made by the stimulus rat. The free-choice exposure between the two observational testing phases may have provided these or other experiences necessary for an effect of another rat's choices to be revealed in the observational testing procedure we used. Additional empirical work will be necessary to determine whether this is the case.

In any case, the performance of rats in the different condition during the second observational testing phase replicated the basic effect found in Experiments 1 and 2. It did so under conditions that rule out involvement of odor or other intramaze cues and require that information be preserved across a temporal gap between the trial phase in which the stimulus rat makes its choices and the trial phase in which the subject rat makes its choices. Thus, these data confirm the use of working memory for the spatial choices made by another rat.

The difference between the performance of rats that had been in the same and different conditions is of interest because it suggests that despite the absence of evidence of social influence during the first observational testing phase, the different contingencies between the visits of another rat and the location of food in that phase did have an effect. Rats in the two conditions were treated identically during the free-choice training and Observational Testing II phases of the experiment. In particular, they all found food only in locations not visited by the other rat during those phases of the experiment. Thus, the difference in contingencies between the other rat's visits and the location of food arranged during Observational Testing I did have an effect. However, that effect was 
latent without the common experience, provided during the freechoice phase of the experiment, of food being absent in locations visited by the other rat.

\section{General Discussion}

Previous work from a variety of perspectives makes it clear that many animals discriminate aspects of conspecifics' behavior and are influenced by it. The results of the present experiments show that the choices made by another rat can be coded in spatial working memory, just as are choices made by the rat itself. The tendency to avoid locations previously visited by another rat was found repeatedly in these experiments, and explanations in terms of odor or other intramaze cues were ruled out by the procedures used in Experiments 1 and 3. Thus, these experiments demonstrate the existence of working memory with social content and provide a technique to isolate such memory.

Although choices are controlled by working memory for the location of choices made by another rat, the extent of that control is not nearly as large as the well-studied ability of rats to avoid locations previously chosen by the rat itself, at least when a forced-choice procedure including a short delay is used. The working memory used to avoid revisits has a duration on the order of several hours (Beatty \& Shavalia, 1980b) and has been shown to be quite resistant to interference (Beatty \& Shavalia, 1980a; Cook \& Brown, 1985). The short delay of a minute (Experiments 2 and 3 ) or two (Experiment 1) following observation of the other rat making choices supported a reliable tendency to avoid the locations chosen by the other rat (though the tendency was modest in magnitude). Furthermore, the effect was revealed by a measure of the order in which locations were visited rather than the more common measures that involve simply whether a location was visited. It seems apparent that the former measure is more sensitive to differences in spatial choice tendencies.

Clearly, a focus of future research should be determination of differences in the information encoded and stored in memory about the subject's own behavior and the behavior of others. The spatial choices made by another rat may serve as a special kind of stimulus that has priority as a cue to the location of food when subsequent choices are made. Alternatively, the other rat may be functioning like any other physical stimulus that might be associated with the unbaited (or baited) locations in the maze. Does another rat have special status as a spatial cue for the location of food? The results of our experiments do not provide an answer to this question.

These results raise other questions about the influence of another rat's behavior on spatial choices. It is unclear why the location most recently visited by the other rat elicits choice of that location whereas locations visited more remotely by the other rat are avoided. The latter tendency occurred when the choices made by the other rat were separated from the choices of the focal rat by an explicit trial interruption and temporal gap (the forced-choice phase of Experiment 1 and the observation test procedure of Experiments 2 and 3) and also when the choices made by the other rat were separated from the choices of the focal rat only by more recent choices made by the other rat. As suggested above, it appears that the critical factor is the physical presence of the other rat. When the other rat may still be physically present near its previous choice, the tendency to visit that location is increased, but when memory is required to code the location of the other rat's choices, avoidance of those locations results. However, the explanation for this difference is unclear. Following familiar conspecifics when there is no other information about the location of food is thought to be common in natural foraging behavior (Galef \& Giraldeau, 2001), and perhaps this effect is elicited by the physical presence of the foraging partner at the same time that contingencies between the other rat's behavior and the location of food are effective via memory.

The comparison of contingencies between visits by the other rat and reinforcement (Experiment 3) was intended to determine whether such tendencies are dependent on the social foraging experiences of the rat. The lack of difference between rats in the same and different conditions during the first observational test phase of that experiment suggests that such experiences play little, if any, role. On the other hand, the results of that experiment may instead indicate that physical contact between the two rats is a necessary part of the social interaction that supports social working memory. In the first phase of observational testing, the rats had very minimal physical contact with each other and did not influence each other's choices. After the physical contact provided by the free-choice trials, rats in the different condition were affected by the choices made by the other rat. Rats in the same condition were not. As discussed above, this finding suggests that the difference in the contingencies during the first phase of observational testing had an effect, even though no differences were revealed by performance at the time. An understanding of the conditions that determine the nature of the influence of another rat's visit to a location will require additional empirical work.

The ability to remember a set of locations visited by a familiar conspecific may be important in supporting a variety of social influences on natural foraging behavior, most obviously determination of food location. More generally, memory for the behavior of others is likely to be a necessary element for a variety of social learning processes (Galef et al., 2005; Zentall \& Galef, 1988). The present experiments provide a basic demonstration that short-term working memory for locations defined by a social stimulus can be isolated.

\section{References}

Barnard, C. J., \& Sibly, R. M. (1981). Producers and scroungers: A general model and its application to captive flocks of house sparrows. Animal Behaviour, 29, 543-555.

Beatty, W. W., \& Shavalia, D. A. (1980a). Rat spatial memory: Resistance to retroactive interference at long retention intervals. Animal Learning \& Behavior, 8, 550-552.

Beatty, W. W., \& Shavalia, D. A. (1980b). Spatial memory in rats: Time course of working memory and effects of anesthetics. Behavioral and Neural Biology, 28, 454-462.

Berdoy, M. (2002). The laboratory rat: A natural history [Video recording]. (Available from www.ratlife.org)

Bond, A. B., Cook, R. G., \& Lamb, M. R. (1982). Spatial memory and the performance of rats and pigeons in the radial-arm maze. Animal Learning \& Behavior, 9, 575-580.

Brown, M. F., \& Drew, M. R. (1998). Exposure to spatial cues facilitates visual discrimination but not spatial guidance. Learning and Motivation, 29, 367-382.

Brown, M. F., Farley, R., Solodky, H., \& Bachrach, R. (2005, March). Oh, the places you've been! Social memories for spatial location. Presentation at the International Conference on Comparative Cognition, Mel- 
bourne Beach, FL. [Abstract available at www.comparativecognition .org/pco3]

Brown, M. F., \& Moore, J. A. (1997). In the dark: II. Spatial choice when access to extrinsic spatial cues is eliminated. Animal Learning \& Behavior, 25, 335-346.

Brown, M. F., Rish, P. A., VonCulin, J. E., \& Edberg, J. A. (1993). Spatial guidance of choice behavior in the radial-arm maze. Journal of Experimental Psychology: Animal Behavior Processes, 19, 195-214.

Brown, M. F., \& Terrinoni, M. (1996). Control of choice by the spatial configuration of goals. Journal of Experimental Psychology: Animal Behavior Processes, 22, 438-446.

Cook, R. G., \& Brown, M. F. (1985). Retroactive interference in rat radial maze performance: The role of point of delay interpolation and the similarity and amount of interpolated material. Animal Learning \& Behavior, 13, 116-120.

Cook, R. G., Brown, M. F., \& Riley, D. A. (1985). Flexible memory processing by rats: Use of prospective and retrospective information in the radial maze. Journal of Experimental Psychology: Animal Behavior Processes, 11, 453-469.

DiMattia, B. V., \& Kesner, R. P. (1984). Serial position curves in rats: Automatic versus effortful information processing. Journal of Experimental Psychology: Animal Behavior Processes, 10, 557-563.

Fretwell, S., \& Lucas, H. (1970). On territorial behavior and other factors influencing habitat distribution in birds: I. Theoretical development. Acta Biotheoretica, 19, 16-36.

Galef, B. G. (1989). Enduring social enhancement of rats' preferences for the palatable and the piquant. Appetite, 13, 81-92.

Galef, B. G., \& Giraldeau, L. (2001). Social influences on foraging in vertebrates: Causal mechanisms and adaptive functions. Animal Behaviour, 61, 3-15.

Galef, B. G., \& Laland, K. N. (2005). Social learning in animals: Empirical studies and theoretical models. Bioscience, 55, 489-499.

Galef, B. G., Lee, W. Y., \& Whiskin, E. E. (2005). Lack of interference in long-term memory for socially learned food preferences in rats (Rattus norvegicus). Journal of Comparative Psychology, 119, 131-135.

Galef, B. G., \& Whiskin, E. E. (2003). Preference for novel flavors in adult Norway rats (Rattus norvegicus). Journal of Comparative Psychology, $117,96-100$

Galef, B. G., \& Wigmore, S. W. (1983). Transfer of information concerning distant foods: A laboratory investigation of the "information-centre" hypothesis. Animal Behaviour, 31, 748-758.

Gallese, V., Fadiga, L., Fogassi, L., \& Rizzolatti, G. (1996). Action recognition in the premotor cortex. Brain, 119, 593-609.

Giraldeau, L. (2000). Social foraging theory. Princeton, NJ: Princeton University Press.

Heyes, C. M., \& Galef, B. G. (1996). Social learning in animals: The roots of culture. San Diego, CA: Academic Press.

Kesner, R. P., Chiba, A. A., \& Jackson-Smith, P. (1994). Rats do show primacy and recency effects in memory for lists of spatial locations: A reply to Gaffan. Animal Learning \& Behavior, 22, 214-218.
Laland, K. R., \& Plotkin, H. C. (1990). Social learning and social transmission of digging for buried food in Norway rats. Animal Learning \& Behavior, 18, 246-251.

Laland, K. R., \& Plotkin, H. C. (1993). Social transmission of food preferences among Norway rats by marking of food sites and gustatory contact. Animal Learning \& Behavior, 21, 35-41.

Lefebvre, L., \& Giraldeau, L. (1996). Is social learning an adaptive specialization? In C. M. Heyes \& B. G. Galef (Eds.), Social learning in animals: The roots of culture (pp. 107-124). San Diego, CA: Academic Press.

Lefebvre, L., Whittle, P. L. E., \& Finkelstein, A. (1997). Feeding innovations and forebrain size in birds. Animal Behaviour, 53, 549-560.

McQuoid, L. M., \& Galef, B. G. (1992). Social influences of feeding site selection by Burmese fowl Gallus gallus. Journal of Comparative Psychology, 106, 137-141.

Olton, D. S. (1978). Characteristics of spatial memory. In S. H. Hulse, H. Fowler, \& W. K. Honig (Eds.), Cognitive processes in animal behavior (pp. 341-374). Hillsdale, NJ: Erlbaum.

Olton, D. S., \& Collison, C. (1979). Intramaze cues and odor trials fail to direct choice behavior on an elevated maze. Animal Learning \& Behavior, 7, 221-223.

Olton, D. S., \& Samuelson, R. J. (1976). Remembrance of places passed: Spatial memory in rats. Journal of Experimental Psychology: Animal Behavior Processes, 2, 97-116.

Olton, D. S., \& Schlosberg, P. (1978). Food search strategies in young rats: Win-shift predominates over win-stay. Journal of Comparative and Physiological Psychology, 92, 609-618.

Suzuki, S., Augerinos, G., \& Black, A. H. (1980). Stimulus control of spatial behavior on the eight-arm radial maze. Learning and Motivation 11, 1-18.

Timberlake, W. (2002). Niche-related learning in laboratory paradigms: The case of maze behavior in Norway rats. Behavioural Brain Research, 134, 355-374

Timberlake, W., \& Hoffman, C. M. (2002). How does the ecological foraging behavior of desert kangaroo rats (Dipodomys deserti) relate to their behavior on radial mazes? Animal Learning \& Behavior, 30, 342-354.

Timberlake, W., \& White, W. (1990). Winning isn't everything: Rats need only food deprivation and not food reward to traverse a radial arm maze efficiently. Learning and Motivation, 21, 153-163.

Zentall, T. R. (2003). Imitation by animals: How do they do it? Current Directions in Psychological Science, 12, 91-95.

Zentall, T. R., \& Galef, B. G. (1988). Social learning: Psychological and biological perspectives. Hillsdale, NJ: Erlbaum.

Zoladek, L., \& Roberts, W. A. (1978). The sensory basis of spatial memory in the rat. Animal Learning \& Behavior, 6, 77-81.

Received August 29, 2006

Revision received January 5, 2007

Accepted January 9, 2007 